\title{
DIME CÓMO EVALÚAS Y TE DIRÉ QUÉ TIPO DE PROFESIONAL Y DE PERSONA ERES
}

TELL ME HOW YOU EVALUATE AND I WILL TELL YOU THE TYPE OF PROFESSIONAL AND PERSON YOU ARE

Miguel Angel Santos Guerra Especialista en Didáctica y Organización Escolar Universidad de Málaga arrebol@uma.es

Resumen: La evaluación es un fenómeno que permite poner sobre el tapete todas nuestras concepciones. Más que un proceso de naturaleza técnica y aséptica es una actividad penetrada de dimensiones psicológicas, políticas y morales. Por el modo de practicar la evaluación podríamos llegar a las concepciones que tiene el profesional que la practica sobre la sociedad, las instituciones de enseñanza, el aprendizaje y la comunicación interpersonal...

Palabras claves: evaluación, disposiciones legales, supervisión institucional, presión social, condiciones órganizativas, evaluador, prácticas evaluativas.

Abstract: Evaluation brings forth, in itself, all our conceptions. In fact, more than an aseptic and technical process it is an activity penetrated by psychological political and moral dimensions. The conceptions a professional has about society, educational institutions teaching and learning so as interpersonal communication, can be inferred through the way he practices evaluation.

Key words: Evaluation, legal regulations, institutional supervision social pressures, organizational conditions, evaluator, evaluative practices.

\section{INTRODUCCIÓN}

Antes de abordar el análisis de esa epifanía que propicia la evaluación quiero plantear cuatro cuestiones preliminares referidas a este complejo fenómeno que condiciona los procesos de aprendizaje y penetra el tapiz de las relaciones que se establecen entre evaluadores, entre evaluados y entre los integrantes de los dos grupos.

Aclaremos, en primer lugar, que la evaluación que se realiza en las instituciones no tiene lugar en una campana de cristal, sino que está condicionada por diversos agentes que inciden sobre ella. Ninguno de los profesionales que trabaja en una institución haría el mismo tipo de evaluación en el caso de que fuese responsable de una actividad particular con un aprendiz al que estuviese tutorizando. El profesor actúa en un contexto que condiciona su práctica de la evaluación. Tiene que evaluar a un número a veces desmesurado de alumnos, entregar calificaciones con unos terminales predeterminados, debe hacerlo en un momento preciso, en unas actas que le son entregadas por los servicios de Secretaría... 
Clasificaré y comentaré, brevemente, a continuación algunos factores que regulan la actividad evaluativa.

\section{FACTORES QUE CONDICIONAN LA EVALUACIÓN}

Prescripciones legales: la evaluación está condicionada por disposiciones legales que la inspiran y la regulan. Estas disposiciones están penetradas de una filosofía que da sentido a la forma de practicar la evaluación. Además, unifican los momentos, la nomenclatura y los contenidos de la evaluación. El profesional no puede hacer la evaluación de la manera que se le antoje, en el momento que quiera, con la nomenclatura que considere oportuna y sobre los aspectos que se le antojen.

Supervisiones institucionales: la forma de hacer la evaluación está supervisada por diversos agentes que velan por el fiel cumplimiento de la norma. Es cierto que cada profesional realiza la evaluación desde una perspectiva particular y con una actitud determinada, pero nadie le libra de seguir las pautas reguladoras. Puede tener un criterio contrario a la norma, pero se ve obligado a cumplirla.

Presiones sociales: la evaluación que realizan los profesionales en las instituciones está sometida a presiones de diverso tipo. Por una parte, al tener el conocimiento un valor de cambio (además del valor de uso, que lo convierte en interesante, práctico o motivador), la calificación que obtiene el evaluado se convierte en un salvoconducto cultural. La familia del alumno se interesa por los resultados de la evaluación obtenidos por sus hijos (frecuentemente, sólo por eso). Por otra parte, la comparación entre las calificaciones obtenidas hace que los evaluados se vean clasificados por los resultados del proceso. La sociedad no es ajena a los resultados de esa clasificación que compara y jerarquiza. Las calificaciones obtenidas y reflejadas en el expediente académico acompañan al estudiante durante toda su trayectoria profesional.

Al comenzar un curso les pedí a mis alumnos que respondieran por escrito a esta cuestión: ¿cómo me defraudaría mi profesor durante el curso? Yo les escribí, también un texto sobre la interrogación recíproca: ¿cómo me defraudarían mis alumnos durante el curso? Cuando leímos nuestros textos respectivos, les dije, entre otras muchas cosas, que me defraudarían si les viese tan interesados por la calificación que el aprendizaje fuese una cuestión marginal 0 anecdótica. Alguien me dijo que en todos los procesos de selección se tenía en cuenta el expediente académico. ¿Cómo no obsesionarse por las calificaciones? Tuvimos que hacer una comisión mixta (de alumnos y profesor) para respondernos a esta otra cuestión, en absoluto baladí: ¿cómo nos defrauda el sistema a ambos? 
Condiciones organizativas: la evaluación se realiza con unos condicionantes determinados como son el tiempo disponible, el grupo de personas evaluadas, el estímulo profesional consiguiente, la tradición institucional, las exigencias del curriculum, las técnicas disponibles, la formación recibida, la cultura organizativa...

En segundo lugar, es preciso reseñar que la evaluación tiene, entre otros, dos componentes básicos: uno es el de comprobación de los aprendizajes realizados. Este primer componente, en sí, es ya muy problemático (Santos Guerra, 1998). No resulta fácil saber cuándo y cómo ha realizado el alumno los aprendizajes pretendidos. Pero hay otro componente de la evaluación que es tenido muy poco en cuenta y que no es menos importante: el de explicación 0 atribución. En efecto, la evaluación viene a decir implícitamente que cuando el aprendizaje no se produce se debe a la exclusiva responsabilidad del que aprende: es torpe, es vago, está mal preparado, tiene poca base, tiene pocos medios, tiene malas influencias, no tiene ayudas, tiene distractores importantes, está inmerso en problemas, no tiene suficiente motivación, no domina las técnicas de estudio necesarias, se pone nervioso al realizar las pruebas...

Obsérvese que pocas veces se responsabiliza a la institución 0 a los docentes del fracaso que tienen los alumnos aunque, en algunas ocasiones sea evidente su influencia en el hecho. Pienso, por ejemplo, en el caso de dos profesores que imparten cursos paralelos, de la misma asignatura, en el mismo centro y cuyos alumnos han sido divididos en dos grupos, partidos por la letra L Los que forman el grupo de la A a la L obtienen resultados satisfactorios y los de la L a la Z fracasan con estrépito. ¿No se puede atribuir nada de los resultados a la actuación de los docentes? ¿Hay que pensar que los alumnos más torpes o más perezosos se agrupan por las iniciales de sus apellidos? La explicación se hace más inquietante si el hecho se repite año tras año. Sin embargo, cada alumno se hará cargo de su fracaso asumiendo toda la responsabilidad y todas las consecuencias del mismo.

En tercer lugar, es conveniente recordar, como ya apuntaba más arriba, que el conocimiento académico tiene un doble valor. Por una parte, tiene valor de uso (es decir, es útil, tiene sentido, posee relevancia y significación, despierta interés, genera motivación...) y por otra parte tiene valor de cambio (es decir, se puede canjear por una calificación, por una nota). Cuando predomina el valor de uso, lo que importa de verdad es el aprendizaje. Cuando tiene una gran incidencia el valor de cambio es porque lo único que de verdad importa es aprobar.

Me pregunto cada día: ¿cuántos de mis alumnos estarán aquí por el deseo y el gusto de aprender o por el interés intrínseco de lo que aprende y cuántos por la imperiosa necesidad de obtener un certificado. Ironiza Gelner sobre los títulos académicos con una divertida anécdota. Dice que por las 
afueras de la ciudad de Edimburgo paseaba un individuo excéntrico que se entretenía en preguntar a la gente:

- ¿Usted está bien de la cabeza?

Las personas solían contestar desconcertadas. pero seguras, que sí, que estaban cuerdas. Pero él seguía con su interrogatorio:

- ¿Me lo puede acreditar?

La respuesta de los interpelados se cargaba de asombro. No sabían cómo podía acreditarse, así de pronto, esa respuesta afirmativa. Pero él decía:

- Pues yo sí lo puedo acreditar de forma inequívoca.

Y sacaba de su cartera un documento que decía en su cabecera: Certificado de alta del manicomio.

Es decir, que es el título y solamente el título quien acredita, en una sociedad, que se han adquirido determinados saberes 0 de que se poseen ciertas destrezas. De ahí su importancia.

Es necesario hacer hincapié en el papel que desempeña la evaluación dentro del curriculum. De alguna manera condiciona todo el proceso de enseñanza/aprendizaje. Cuando el énfasis se acaba situando en la consecución de buenas calificaciones es porque el interés que tiene el conocimiento es, cuando menos, secundario. No tienen por qué ser antagónicos el valor de uso y el valor de cambio, pero la práctica pervertida puede exclusivizar el interés por las calificaciones. Se puede observar con preocupación cómo algunos alumnos -incluso, grupos enteros- forcejean con el profesorado para que se vean menos temas, para que el programa sea más corto, para que sea más fácil aprobar (a costa de que el aprendizaje sea también menor o de que no exista). Se puede comprobar fácilmente cómo los alumnos desean irse pronto de vacaciones, hacer puentes entre días festivos, retrasar el comienzo de las clases... Con tal de que no se amenace el aprobado, todo es aceptable.

En cuarto lugar, deseo advertir que la forma de practicar la evaluación potencia 0 debilita un tipo de operaciones intelectuales u otro según los criterios que fija el evaluador. Si se pide que se jerarquicen por orden de complejidad intelectual las siguientes tareas (todas importantes, todas necesarias) está claro que el orden tendrá carácter descendente. La de menor potencia será memorizar y la de mayor potencia, crear.

MEMORIZAR

APRENDER ALGORITMOS

COMPRENDER

ESTRUCTURAR

COMPARAR

ANALIZAR

ARGUMENTAR 


\section{OPINAR \\ INVESTIGAR \\ CREAR}

Ahora bien, si se observa qué orden ocupan estas tareas en las pruebas y exigencias de la evaluación, probablemente esté invertido el orden. De esta manera, la evaluación estará puesta al servicio de las tareas intelectualmente más pobres. Es decir, la estructura de tareas (Doyle, 1979) está muy vinculada a la naturaleza del fenómeno evaluador.

La complejidad que encierra el proceso de evaluación es tan grande que resulta sorprendente el reduccionismo con el que frecuentemente se practica en el marco de las instituciones. Una de las causas de la simplicidad es un reduccionismo lingüístico que confunde evaluación con calificación. En efecto, poner una nota no es un proceso de evaluación, como no lo es la simple medición de la longitud de una mesa. Otra causa de la simplificación proviene de la selección de los contenidos evaluables. ¿Por qué sólo conocimientos y destrezas?, ppor qué no actitudes y valores? Una tercera causa es la pretendida tecnificación que despoja a la evaluación de dimensiones más complejas como la justicia, la diversidad, la emotividad...

\section{LA EVALUACIÓN PERMITE DEVELAR LAS CONCEPCIONES DEL EVALUADOR}

La forma de entender y de practicar la evaluación permite deducir cuáles son las teorías sobre las que ésta se sustenta. No es cierto que los prácticos no tengan teorías, otra cosa es que éstas sean explícitas. ¿Qué tipo de concepciones permite develar la evaluación?

a.- Sobre la naturaleza de la inteligencia: si se piensa que la inteligencia es algo dado de una vez para siempre a las personas, que no está condicionada por factores sociales, culturales, lingüísticos, económicos..., será más fácil clasificar a los sujetos en grupos de fracaso o de éxito. Si, por el contrario, se piensa que la inteligencia es algo que se construye, que depende de influjos culturales, que tiene la potencialidad de desarrollarse y enriquecerse, la evaluación estará más abierta a la flexibilidad.

Hay evaluadores que clasifican y etiquetan a los evaluados atribuyéndoles unas capacidades inamovibles que, de alguna manera, condicionan las expectativas y predisponen al fracaso 0 al éxito. La política de etiquetado, como certeramente explica Michael Apple (1986) no se realiza de manera casual:

"Una gran parte de la literatura de etiquetado de los niños en la escuela descansa en una rama particular del idealismo. Es decir, supone que las 
identidades de los alumnos son creadas totalmente por las percepciones que los profesores tienen de los estudiantes en el aula. Sin embargo, no es simplemente una cuestión de que la conciencia de los profesores crea la conciencia de los niños: por ejemplo, un profesor concibe a un estudiante como realmente torpe y, en consecuencia, el niño se vuelve realmente, aunque con toda seguridad hay en esto algo de verdad. Además de eso se hallan también profundamente implicadas las expectativas y circunstancias materiales objetivas que forman y rodean el entorno escolar" (Apple, 1986:184-185).

Es decir, que es más fácil que se apliquen etiquetas de bajo potencial a personas de clases menos pudientes, culturalmente depauperadas 0 de escasa expectativa intelectual.

b.- Sobre el proceso de enseñanza/aprendizaje: la forma de evaluar devela el concepto que el docente tiene de lo que es enseñar y aprender. No sólo de qué es lo que el aprendiz tiene que asimilar sino de la forma en que el profesor puede ayudarle a hacerlo.

Me serviré de dos metáforas para explicitar estas afirmaciones. Primera metáfora: El docente es una persona que posee el conocimiento en un recipiente. Por ejemplo, una botella de agua. El aprendiz es una copa (elemento por completo pasivo) que recibe el agua de manos del docente. La evaluación consistirá en preguntarse por el agua que contiene esa copa. Si no hay agua dentro de ella es porque no estaba debajo del chorro de agua que se vertía de la botella. Segunda metáfora: el docente en un experto en buscar manantiales de agua. La tarea docente consiste en enseñar al aprendiz a localizar por sí mismo manantiales de agua. La evaluación consistirá, en este caso, en comprobar si el aprendiz es capaz de buscar por sí mismo fuentes de agua y de saber si ésta es salubre o está contaminada.

Cuando se concibe la enseñanza como un proceso mecánico de transmisión de conocimientos, linealmente estructurado (sólo puede aprender el alumno, sólo puede aprender del profesor...), la evaluación consistirá en comprobar si, efectivamente, el aprendizaje se ha producido.

¿Se potencia, desde esa concepción, el aprendizaje autónomo, la búsqueda de conocimiento independiente? ¿Se practica el aprendizaje compartido, el trabajo en grupo, la búsqueda colegiada? ¿Se piensa, desde esta concepción, en los efectos secundarios del aprendizaje? Evidentemente, no. De ahí el hecho, tantas veces denunciado por los alumnos de que han aprendido algo o mucho de algunos profesores, pero que han aprendido simultáneamente a odiar el aprendizaje y, concretamente, el aprendizaje de esa materia.

c.- Sobre la naturaleza de la profesión: quien concibe al docente como un profesional dedicado a realizar prácticas asépticas, despojadas de dimen- 
siones psicológicas y sociales, practicará una evaluación centrada en la adquisición de datos y en el dominio de competencias.

$\mathrm{Si}$ alguien entiende que el docente es una persona encargada de introducir en la cabeza del aprendiz una serie de datos, conceptos, teorías y destrezas, no se planteará ninguna cuestión sobre las exigencias morales, las relaciones interpersonales, las dinámicas sociales que conlleva una forma distinta de entender la profesión.

\section{LA EVALUACIÓN PERMITE DEVELAR LAS ACTITUDES DEL EVALUADOR}

a.- Hacia sí mismo: si uno tiene la conciencia de que parte del aprendizaje depende de la forma en que se ha enseñado, mantendrá la humildad de reconocer como suyo parte del fracaso de los que aprenden. No pensará que si el aprendizaje no se produce es por responsabilidad exclusiva del aprendiz.

Quien se vive a sí mismo desde la displicencia, desde una actitud masoquista, será probable que aborde la evaluación desde posiciones sádicas. Es más que probable que convierta la evaluación en una instancia de poder que lo parapeta ante interpelaciones inquisidoras.

La evaluación no se utiliza como un instrumento de aprendizaje para el que enseña (Santos Guerra, 2001), sino como un simple modo de comprobación del esfuerzo realizado por el que aprende.

b.- Hacia los evaluados: como la evaluación encierra poder, hay quien la utiliza como un arma con la que amenaza y castiga. Lejos de emplearla como un estímulo, le sirve como instrumento de opresión. "Ten cuidado con lo dices o con lo que haces, porque te puedo suspender", dice el evaluador que se atrinchera detrás de su capacidad de sentenciar.

Este poder es a veces ejercido por el evaluador y, a veces, es vivido por el evaluado que practica un servilismo interesado que dará frutos en el momento de las calificaciones.

Este clima adultera la convivencia y genera una dinámica de falsedades y de perversión institucional. El que evalúa no recibe críticas de los evaluados por el temor a que se produzcan represalias o castigos, más 0 menos velados. A sus espaldas dirá el evaluado todo lo que piensa y lo que siente, pero esa información nunca se podrá utilizar para reflexionar o para el mejorar el proceso, ya que ni siquiera es conocida.

Una forma perniciosa de poder es aquella que consiste en la imposición de la verdad. La defición (jerárquica) de verdad como "aquello que la auto- 
ridad dice que es verdad" tiene mucho que ver con la práctica de la evaluación, ya que ésta ha consistido esencialmente en dar respuestas que el evaluador considera como válidas. ¿Quién no ha tenido que renunciar a sus propios criterios para acomodarse a los de un evaluador estricto?

Los alumnos renuncian, con mucha frecuencia, al derecho que la ley les confiere de hacer reclamaciones sobre la calificación obtenida, ya que consideran que pueden salir malparados. Y, en caso de acudir a comisiones evaluadoras alternativas, tienen la sospecha de que los profesores adoptarán una actutud gremialista que defienda los criterios de un compañero.

c.- Hacia los colegas: la evaluación suele ser una práctica individualista en un doble sentido. En primer lugar porque cada evaluador la practica según sus peculiares criterios. En segundo lugar porque cada evaluado tiene que afrontar de manera individualista, la comprobación de su aprendizaje. Hasta cuando se hacen trabajos de grupo el evaluador se muestra inquisitivo para saber qué hizo cada uno de los integrantes del mismo. Es grupal el trabajo, pero la evaluación sigue siendo estrictamente individual.

Una práctica individualista de la evaluación deja en evidencia la forma de actuar de otros compañeros. Detrás de esa forma de actuación (cada uno a su tarea, "cada maestrillo con sus actillas") subyace una concepción egocéntrica del proceso de enseñanza y aprendizaje. Ni siquiera lleva a la reflexión el comprobar las significativas discrepancias entre los resultados de la evaluación realizada por compañeros. Si en segundo curso obtienen mejores calificaciones que en primero, idónde adquirieron la base necesaria? Si van bien en todas las asignaturas y sólo mal en la mía, ¿qué sucede con mi forma de evaluar? Si con todos los colegas obtienen aprobados, por qué conmigo todos obtienen sobresaliente?

En realidad, sólo importa "mi" asignatura, "mi" alumno, "mi" evaluación". Las preguntas de más calado ni siquiera se plantean. No aparecen interrogantes del siguiente tipo: ¿cómo salen formados los aprendices?, ¿qué tipo de profesional buscamos?, ¿qué exigencias tiene la práctica y cómo las satisfacemos?, ¿cómo se hace la simbiosis teoría/práctica?, ¿cómo se forman las actitudes?...

\section{LA EVALUACIÓN PERMITE DEVELAR LOS PRINCIPIOS ÉTICOS DEL EVALUADOR}

La evaluación no es sólo un procedimiento mecánico que conduce a una calificación sino que encierra, por el contrario, dimensiones éticas de naturaleza nada desdeñables. ¿Se ha pensado lo suficiente sobre los sentimientos que se mueven -tanto de profesores como de alumnos- en torno al 
fenómeno de la evaluación? ¿Se ha planteado con profundidad lo que sucede con los componentes morales del proceso evaluador?

Veamos algunos de los principios que, a través de la forma de hacer la evaluación, quedan de manifiesto y muestran cómo se carga de sentido moral todo el proceso.

a.- Sobre la estructura y la dinámica social: quien concibe la realidad social como un entramado fatalista de jerarquías, no será sensible a los perjuicios y a las dificultades que los más desfavorecidos tienen para acceder al conocimiento. Unos tienen muchos medios psicológicos (expectativas, estímulos, motivaciones, facilitadores...) y materiales (dinero, libros, internet...) y parten con ventaja en esa lucha por conseguir los mejores puestos. La evaluación tiene que ver con la racionalidad pero también con la justicia.

Una determinada forma de practicar la evaluación, eminentemente tecnicista, pretendidamente aséptica, obsesionada con mediciones estandanizadas, es poco sensible a las diferencias económicas, sociales y culturales.

b.- Sobre finalidad de las instituciones: quien piensa que las instituciones han de convertirse en filtros sociales, que han de clasificar a los sujetos en función de sus capacidades, hará una evaluación tendente a establecer rigurosas competiciones clasificadoras. Si, por el contranio, alguien piensa que las instituciones tienen la tarea de ayudar a que las personas crezcan y lleguen a sus máximas posibilidades, hará una evaluación más individualizada y sensible a las diferencias.

Estas concepciones están condicionadas, entre otros muchos factores, por la cultura que invade la sociedad y las mismas instituciones (Pérez Gómez, 1998), que no pueden quedarse al margen del clima cultural en el que se mueven. Por eso, resulta fácil dejarse arrastrar hoy día por las corrientes neoliberales que sumergen a las instituciones en una dinámica de competitividad, de obsesion por la eficacia y por el éxito individualista de sus integrantes.

c.- Sobre las exigencias morales de la profesión: Las profesiones en las que se produce una interacción profunda entre las personas se sustentan sobre unas exigencias morales que no pueden ser olvidadas.

Si un docente, sólo se preocupa de la dimensión técnica del aprendizaje, del progreso intelectual, del éxito académico del aprendiz, estará olvidando los principios básicos de la relación humana y de un ejercicio profesional responsable. 


\section{ALGUNAS CONCLUSIONES ENCAMINADAS A MEJORAR LA PRÁCTICA}

Podría darse la vuelta al título y al contenido de este texto. Todas las concepciones, las actitudes y los principios del profesional conducen a una práctica evaluadora determinada. A su vez, la práctica permite descubrir las concepciones, las actitudes y los principios que la sustentan. De ahí mi interés por cerrar estas líneas con sugerencias para mejorar la práctica de la evaluación.

\section{ES PRECISO PONER EN TELA DE JUICIO LAS PRÁCTICAS EVALUADORAS}

Si no se somete la práctica a un análisis riguroso que ponga en entredicho el entramado de principios, exigencias y normas, será difícil comprenderla y transformarla.

"El análisis de la evaluación no puede realizarse sin una referencia directa a la acción educativa y al concepto de aprendizaje que resulta de ella... Sería aconsejable, pues, empezar por examinar con ojo crítico las tesis planteadas, para luego, en un segundo momento, considerar nuevas prácticas de evaluación que estarían en mejores condiciones de responder a cada una de las opciones pedagógicas" (Bélair, 2000:13).

Esta revisión constante de las prácticas y de los principios que la sustentan ha de hacerse de forma cooperativa y no solamente individualizada. Compartir los interrogantes, reformular las preguntas, desmontar las paradojas (Santos Guerra, 1999), analizar las repercusiones de un determinado modo de proceder, resulta imprescindible para la mejora de la evaluación.

La evaluación se ha circunscrito a los aprendizajes de los alumnos pero, como es lógico, hay que evaluar también la docencia (Jiménez Jiménez, 1999). Si se evalúa la docencia, será más fácil mejorar los procesos de aprendizaje.

\section{LAS PREGUNTAS HAN DE CONDUCIR A UN PROCESO DE INVESTIGACIÓN RIGUROSO}

Formularse preguntas no lo es todo. Si una vez formulados los interrogantes, respondemos con intuiciones, suposiciones, teonizaciones sin fundamento, no comprenderemos el problema y, además, defenderemos intereses espurios que mantengan el statu quo de nuestras prácticas.

La investigación exige rigor. Ahora bien, hay quien concibe la investigación como un sofisticado y complejo sistema de carácter estadístico que solamente los técnicos dominan. Entiendo por investigación un proceso de análisis de la realidad que se apoya en evidencias y en argumentaciones 
cargadas de rigor. Digo esto porque algunos docentes entienden que la investigación educativa es competencia exclusiva de los expertos.

Cuando un docente se hace preguntas sobre la evaluación y responde a las mismas apoyándose en evidencias, en hechos rigurosos, en argumentos consistentes, está investigando.

\section{DESDE LA COMPRENSIÓN QUE GENERA LA INVESTIGACIÓN ES PRECISO TOMAR DECISIONES DE MEJORA}

El sentido educativo que ha de tener la investigación exige que ésta se ponga al servicio de la mejora de las prácticas y que no sirva sólo para llenar los anaqueles de tomos cuajados de hermosas y consistentes teorías.

El conocimiento ha de estar centrado en la acción evaluadora y encaminado a la mejora de la misma. Entendiendo por mejora no sólo la multiplicación de sus exigencias técnicas sino la racionalidad de la práctica y el respeto a la equidad que la educación auténtica exige.

El motor que impulsará la transformación y la mejora de la evaluación que realizan los profesionales es la comprensión que genera la investigación que realizan.

\section{ES PRECISO EXIGIR LAS CONDICIONES NECESARIAS PARA QUE IA MEJORA LEGUE A BUEN TÉRMINO}

La mejora no depende sólo de las concepciones, de las actitudes y de los principios que inspiran la práctica de los evaluadores. Hace falta transformar las situaciones en las que se desarrolla la práctica. Esas condiciones no se modifican por arte de magia. Es necesario ejercer la crítica y organizar la exigencia de reivindicaciones, ya que no siempre los Administradores están dispuestos a escuchar y a facilitar la optimización de esas situaciones.

Nuestra condición de profesionales y de ciudadanos nos exige un compromiso con la transformación que pasa por mejorar las condiciones que la hagan posible. Para ello hace falta romper el individualismo, desmontar el fatalismo y practicar la valentía cívica, que es una virtud democrática que nos hace ir a causas que de antemano sabemos que están perdidas. 


\section{REFERENCIAS BIBLIOGRÁFICAS}

Álvarez Méndez, J.M. 2000

Apple, M. 1986

Bélair, L.M. 2000

Cano G., E. 1998

Doyle, W. 1979

González Ramírez, T. 2000

House, E. 1984

Jiménez Jiménez, B. 1999

Pérez Gómez, A.I. 1998

Santos Guerra, M. a. 1993

Santos Guerra, M.A. 1998

Santos Guerra, M. A. 1999

Santos Guerra, M.A. 2001

Tombari, M. y Borich, G. 1999
Evaluar para conocer, examinar para excluir. Ed. Morata. Madrid.

Ideología y currículo. Ed. Akal. Madrid.

La evaluación en la acción. Ed. Díada. Sevilla.

Evaluación de la calidad educativa. Ed. La Muralla. Madrid.

Clasroom Tasks and the Students'Abilities. En: Peterson y P.L y Walberg, H.J. (Eds) Research on teaching Concepts, Findings and Implications. McCutchan Publishing Corporation. Berkeley. California.

Evaluación y gestión de la calidad educativa. Un enfoque metodológico. Ed. Aljibe. Archidona.

Evaluación, ética y poder. Ed. Morata. Madrid.

Evaluación de la docencia. En: Jiménez, B. y Otros Evaluación de programas, centros y profesores. Ed. Síntesis. Barcelona.

La cultura escolar en la sociedad neoliberal. Ed. Akal. Madrid.

La evaluación, un proceso de diálogo, comprensión y mejora. Ed. Aljibe. Archidona.

Evaluar es comprender. Ed. Magisterio del Río de la Plata. Buenos Aires.

20 paradojas de la evaluación en la universidad española. Revista electrónica http://www.uva.es/ aufop/aufopweb.htm.

Enseñar o el oficio de aprender. Organización escolar y desarrollo profesional Ed. Homo Sapiens. Rosario.

Authentic Assessment in the Classroom. Merrill, Ohio. 